\title{
Paradigms in Fluorescence Molecular Imaging: Maximizing Measurement of Biological Changes in Disease, Therapeutic Efficacy, and Toxicology/Safety
}

\author{
Jeffrey D. Peterson $\odot$ \\ PerkinElmer, Inc., Discovery \& Analytical Solutions, 68 Elm Street, Hopkinton, MA, 01748, USA
}

\begin{abstract}
Fluorescence molecular imaging (MI) is an important concept in preclinical research that focuses on the visualization of cellular and biological function in a non-invasive fashion to better understand in vivo disease processes and treatment effects. MI differs fundamentally from traditional preclinical imaging strategies in that it generally relies on reporter probes specific for particular targets or pathways that can be used to reveal biological changes in situ, at the site(s) of disease. In contrast, the more established imaging modalities, like magnetic resonance imaging, X-ray, micro X-ray computed tomography, and ultrasound, historically have relied primarily on late-stage anatomical or physiologic changes. The practical application of fluorescence $\mathrm{Ml}$, however, has drifted somewhat from the emphasis on quantifying biology, and based on the publication record, it now appears to include any imaging in which a probe or contrast agent is used to non-invasively acquire in vivo endpoint information. Unfortunately, the mere use of a defined biologically specific probe, in the absence of careful study design, does not guarantee that any useful biological information is actually gained, although often useful endpoint results still can be achieved. This review proposes to add subcategories of MI, termed $\mathrm{Ml}$ biological assessment (or MIBA), that emphasize a focus on obtaining early and clear biological changes associated with disease development, therapeutic efficacy, and drug-induced tissue changes. Proper selection of probes and careful study design are critical for maximizing the non-invasive assessment of in vivo biological changes, and applications of these critical elements are described.
\end{abstract}

Key words: Molecular imaging (MI), Near-infrared fluorescence, Cancer, Inflammation, Toxicology, Biomarker, Molecular imaging biological assessment (MIBA)

\section{Introduction}

Preclinical imaging in academic and pharmaceutical research historically has focused on useful and interpretable physical/ anatomical changes for the assessment of disease progres-

Correspondence to: Jeffrey Peterson; e-mail: jeff.peterson@perkinelmer.com sion, severity, and treatment efficacy. For example, current important imaging readouts include tumor size in oncology, joint space width in osteoarthritis, bone density in osteoporosis, bronchial wall thickening in emphysema, structural abnormalities or lesions in heart, lungs, brain, liver, or gastrointestinal disease, and many others. These and other standard imaging assessments continue to be of importance in preclinical research as well as serving as current gold standard readouts for establishing clinical disease status and 
treatment efficacy. However, these approaches also present significant limitations since they are generally best suited for later detection of overt tissue changes that result from long and complex disease processes rather than on discerning or characterizing disease mechanisms. So, the concept of "Molecular Imaging" (MI), which arose in the mid-1990s, has come along as a growing research discipline with the goal of non-invasively visualizing biological/cellular function in living organisms. This is generally achieved by the use of specific imaging probes designed to target important biomarkers in vivo, and probe quality (e.g., selectivity, biodistribution, signal intensity, and overall in vivo performance) is a critical component of success. Depending on the nature of the readout, the probe/target interaction, and/or the specific biology being assessed, the intensity or pattern of signal in acquired images will change in response to diseaseor drug-induced biological changes at the site of interest. The ability to non-invasively image often subtle biological changes opens up a number of exciting possibilities for pharmaceutical research, including improving our understanding of the biology of disease progression and providing early guidance in understanding therapeutic efficacy. Furthermore, MI can provide better opportunities for objective quantification of biology, improving the interpretation of disease studies.

There are a few different imaging modalities that can be used to varying degrees for preclinical non-invasive MI, including positron emission tomography (PET), single photon emission tomography (SPECT), optical, magnetic resonance imaging (MRI), ultrasound, and micro X-ray computed tomography (microCT). Each modality has its unique strengths and weaknesses and some are more useful for imaging physiologic activity, while others show flexibility in both physiologic and biological imaging. In particular, PET and SPECT have helped to set the paradigm for molecular imaging through techniques that use radiolabeled molecules to monitor biological processes in vivo. As an example, biological changes in glucose metabolism [using 2deoxy-2- $\left[{ }^{18} \mathrm{~F}\right]$ fluoro-D-glucose $\left.\left(\left[{ }^{18} \mathrm{~F}\right] \mathrm{FDG}\right) / \mathrm{PET}\right]$ can be seen early in the course of some diseases, including Huntington's, Alzheimer's disease, and cancer [1-3]. Other imaging modalities have also shown some advances in MI techniques and probes that are now being used in research. These include, for example, targeted and activatable MR probes [4, 5], targeted ultrasound probes [6], and specialized microCT contrast agents [7]. Fluorescence imaging, at least for preclinical use, offers the greatest flexibility in application to molecular imaging, albeit with some limitations based on the physics of light propagation through biological tissues. The stability of fluorescence reporters, simplicity of generating reporter conjugates, the commercial availability of a number of instruments, fluorophores and imaging probes, capabilities in multiplex imaging, the low cost and ease of use of optical imaging systems, and the relatively high-throughput of imaging make fluorescence MI a powerful option for preclinical research. Advances in near- infrared (NIR) fluorescence tomography and algorithms for modeling light propagation through tissue [8-11] have also allowed imaging of fluorescence in $3 \mathrm{D}$ in living mice on instruments like the FMT ${ }^{\circledR} 4000$ and IVIS ${ }^{\circledR}$ SpectrumCT (PerkinElmer, Waltham MA) further improving the overall utility of fluorescence imaging. This review will focus on fluorescence strategies that exemplify non-invasive MI approaches that can differentiate it from standard preclinical and clinical readouts and provide unique biological information on disease progression and therapeutic intervention.

\section{Defining MI in the Context of Biomarker Classifications}

Although MI as a research approach is extremely valuable, the term itself can be confusing, and the range of what is considered MI is often interpreted differently by different researchers. In 2007, the SNM Molecular Imaging Center of Excellence defined MI very well as "the visualization, characterization, and measurement of biological processes at the molecular and cellular levels in humans and other living systems." They further defined this as including twoor three-dimensional imaging, quantification over time, and modalities, such as MR imaging, MR spectroscopy, optical imaging, ultrasound, and others [12]. Based on the published literature, however, the practical definition appears to be more liberal among researchers, and MI appears to mean any type of imaging in which a probe or contrast agent is used to non-invasively acquire in vivo imaging data. Thus, many MI publications include terminal readouts that correlate with non-imaging metrics (e.g., tumor caliper measures, arthritis paw swelling, serum biomarkers, clinical scoring, ex vivo wet tissue weights, histopathology, and many others, depending on the animal model), yet they provide little or no actual biological information beyond the knowledge of the probe's specificity. For example, if you use a fluorescent imaging probe that measures tumor metabolism yet image at a late stage in tumor treatment (i.e., comparing a large, untreated, growing tumor to a small, dying and regressing tumor), are you truly imaging tumor metabolism? Results may provide useful and objective measurements of tumor burden/size; however, are they telling you anything about tumor biology or the biological effects of treatment? It is certainly difficult to justify measurements in dying tissue as providing valuable biological information beyond the actual lack of living biology. On the other hand, some would claim that measurement of blood flow or general physiologic functions should not be included as MI because they do not measure a molecular or cellular process, although others would argue that vascular flow or leakage are biological processes and should be considered as MI strategies.

It is important to note that general success in fluorescence MI is dictated in large part by the quality of the probe(s) used, which must be well-designed and well-validated in vitro and in vivo. This means that many validation studies must be 
performed by probe-developers to understand and characterize probe performance. A protease-activatable probe must be exhaustively profiled in vitro for enzymatic cleavage by the target protease, related family members, and unrelated proteases expected to be at the disease site(s). Cell assays should be performed to understand intracellular $v s$. extracellular activation, and specific protease inhibitors can further confirm specificity. For targeted probes, appropriate cell binding and cell specificity (e.g., comparison to non-expressing cells and/or competition blockade of binding) should be performed by flow cytometry or microscopy with measures of binding affinity where possible. Non-specific in vitro binding interference should be assessed with physiologic levels of albumin or serum to assure that the probe will translate into the in vivo environment. In vivo probe validation, at early stages, is more about characterizing the drug-like properties of the probe than it is about efficacy performance. For all probes, the in vivo biodistribution profile (i.e., major routes/kinetics of sequestration or clearance) must be assessed (and modified by probe structural modification if necessary) to best understand and minimize potential interference with target site imaging. The blood pharmacokinetics should be understood and, ideally, tailored to the type of probe, e.g., a long half-life for activatable probes to allow sufficient time for tissue uptake and cleavage, and a short half-life for high-affinity targeted probes to facilitate early clearance of unbound probe. Specificity should be re-confirmed in in vivo disease models by using either competition (excess unlabeled targeting moiety) or chemical target inhibitors. Sufficient targeted signal should be observed to allow clear and un-subjective definition of the target site and the ability to robustly quantify changes as low as $30 \%$. Accurate patterns of in vivo-delivered tissue localization should be confirmed in ex vivo frozen tissue sections by using labeled antibodies to the same target to compare patterns of microscopic tissue localization. Certain types of probes, like nanoparticles and fluorescent-labeled antibodies, will be generally problematic and will often not perform well in these validation assessments due to issues with poor tissue penetration, long circulation half-lives, high levels of phagocytic cell uptake, and high levels of liver uptake. High-performing and well-characterized probes offer the greatest range of imaging opportunities and provide confidence in the biological interpretations of imaging results, whereas probes with significant performance issues must always be interpreted cautiously, and may necessitate doing your own validation studies prior to use.

Before we consider the overall concept of MI, see Fig. 1, a diagrammatic representation of different biomarker classes to contemplate when selecting an imaging strategy. First, there are disease markers that can be used at various time points in assessing disease progression as compared to normal animals. This includes predictive markers, bioprofiling markers (i.e., biomarkers that characterize different aspects of early disease biology), and diagnostic markers (i.e., biomarkers that align with later-stage clinical disease detection). Second, there are treatment markers that are used to detect differences between diseased animals that are either untreated or undergoing drug treatment. This category includes early assessments of pharmacodynamic (PD) markers (e.g., direct measurement of effects on the drug's specific target/pathway or on an appropriate near downstream target) and predictive efficacy markers (biomarkers of the disease process itself). Late-stage readouts of treatment effects are also possible, including unique in-life biological endpoints that offer deeper biological insight than standard readouts as well as late-stage surrogate markers that correlate with existing "gold standard" clinical readouts. There can also be predictive and surrogate markers for druginduced tissue injury as well, independent of drug efficacy assessment. Lastly, there are clinical endpoints that include a variety of non-MI assessments (depending on the disease) that are clinically/preclinically validated. Some probes can actually serve as one or more of these types of biomarker readouts, depending on the study design, whereas others may be more limited in the scope of their utility.

The majority of fluorescence imaging publications (in which non-invasive imaging is used) focus on diagnostic or surrogate endpoint approaches (MI types 5 and 6). Many of these publications fall under the category of developing and/or characterizing probe performance in detecting overt disease and/or overt treatment efficacy in comparison to gold standard readouts. This is certainly a valuable first step in probe validation, but it is generally the lowest hurdle to pass when comparing, for example, tumor versus regressed tumor, inflammation versus no inflammation, etc. Such an approach will generally provide quantifiable differentiation, yet it may not be ideal if the researcher is trying to gather impactful biological information. In other words, one could expect, with any biomarker showing expression in the disease condition, that "no disease" signal will be low and "high disease" signal will high. For clinical or surrogate treatment markers, any treatment that shows significant efficacy will likely be associated with an overall gross decrease in tumor size or inflammation, yielding a proportional drop in imaging signal intensity. Thus, even though size-normalization strategies can be applied, realistically it becomes difficult to confidently interpret the biology when there are significant differences in size/scope at the site of interest.

Rather than re-defining fluorescence MI to more narrowly cover only those scenarios that provide maximal biological information, as discussed above, it is a useful mental exercise to identify subcategories of MI that can be defined as MI biological assessment (or MIBA). These would be defined as those imaging strategies that can provide, for example, early detection in disease, early detection of therapeutic efficacy, and characterization of different biological effects between mechanistically distinct therapeutic treatments. Predictive and bioprofiling biomarker strategies (MIBA-1) are imaging approaches in which the characteristics or specificity of imaging probes are key to early detection of disease (or to profiling biological changes during disease onset) prior to overt clinical signs. MIBA-1 imaging may require comparison of multiple probes specific for different biological targets and/or ex vivo corroboration 


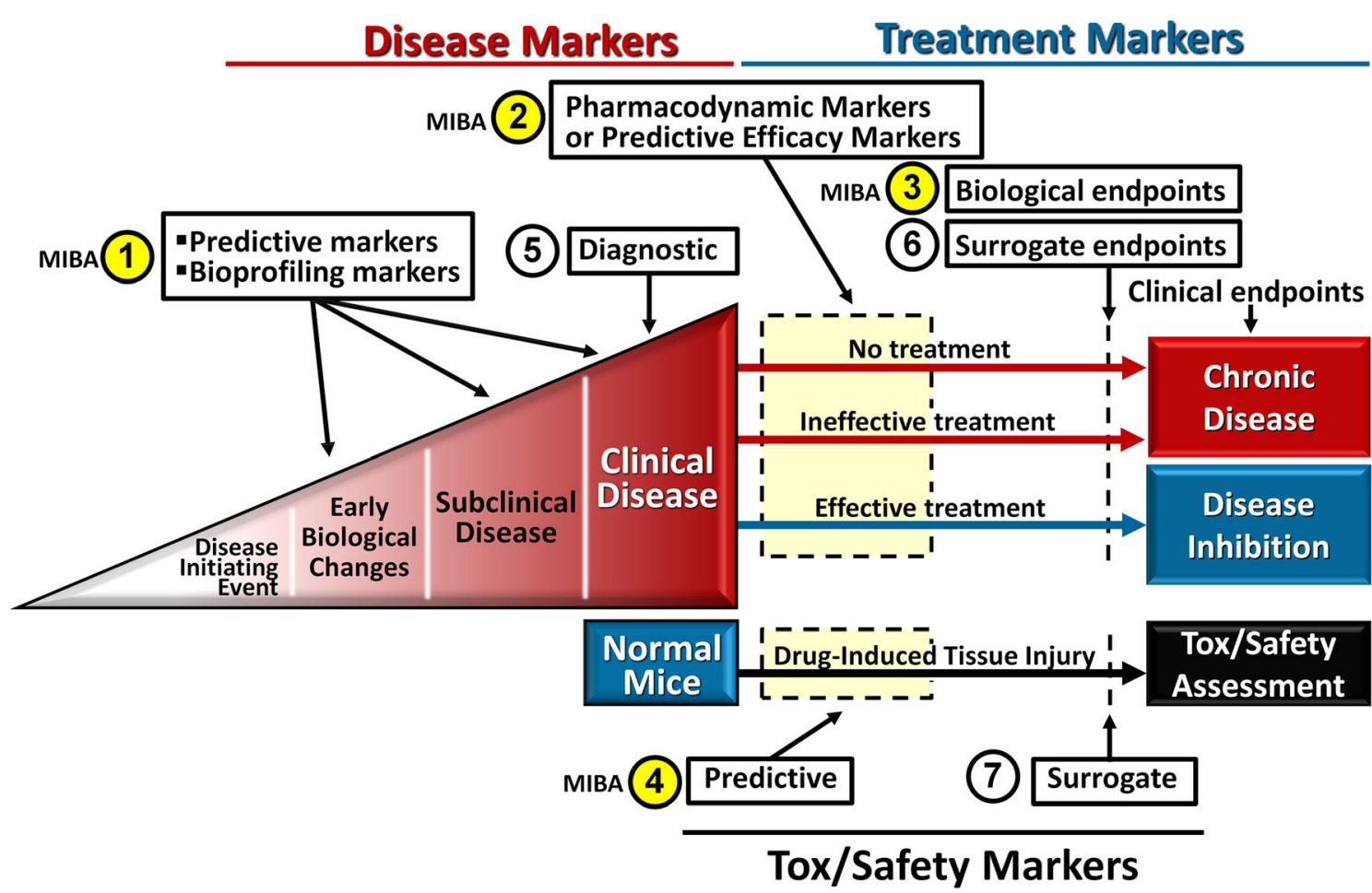

Fig. 1. Diagram representing disease progression and treatment in the context of seven different fluorescent molecular imaging (MI) approaches. Imaging categories 1-4 (yellow-highlighted numbers) indicate MI biological assessment (MIBA) strategies that can maximize acquisition of biological information. Categories 5-7 (white-highlighted numbers) are useful latestage imaging strategies that can provide surrogates for other established, validated readouts but will generally yield less biological information.

of the unique early expression of the particular probe target in order to truly classify as MIBA. PD markers or predictive efficacy markers (MIBA-2) rely on both the selection of appropriate probes as well as using an appropriate study design in order to provide biologically relevant data. Ideally, assessment should be very early post-treatment to best separate treatment-induced biological changes from gross physiological or anatomical changes, and probes should be used to detect biological targets proximal to the mechanism of action or targets downstream of affected pathway(s). Naturally, fluorescence imaging generally requires examination of cell surface or lysosomal/endosomal targets, placing some limitations on possibilities for PD marker approaches for MIBA-2. MIBA-3 includes non-invasive biological endpoint imaging strategies that generate information that is either superior to existing endpoints, adds a new dimension of biological understanding, and/or is predictive of more labor-intensive ex vivo histology or cellular analyses. MIBA-4 is a relatively new application area for fluorescence imaging that is oriented to assessing druginduced, off-target biological changes in tissues that may predict a drug safety concern. Recent publications have suggested MIBA-4 approaches that can detect drug-induced biological changes after a single drug dose $[13,14]$ and in a variety of tissues (including liver and kidneys), that precede tissue changes as detected by histopathology [15]. In this case, the strategy relies on early testing post-drug and the use of probes, or probe combinations, that detect general biological changes associated with inflammation, fibrosis, metabolic changes, and apoptosis.

In summary, the current MI approaches used by many (certainly not all) researchers often favor straightforward latestage readouts, many times underutilizing or undervaluing the potential contribution of well-designed fluorescent MI probes. So, I have outlined MI biological assessment strategies, termed MIBA, that may offer advantages (from the perspective of gaining maximal biological insight) through the application of changes in study design, imaging time points, and careful probe selection. Appropriate use of MIBA strategies should enable earlier decision-making in the drug-development process as well as provide more information regarding disease progression, drug efficacy, and drug safety. Throughout the remainder of this review, I will provide some examples of effective fluorescence MIBA in cancer, inflammation, and toxicology imaging.

\section{MIBA Strategies in Cancer}

Cancer is a complex disease, characterized by changes in a variety of different physiological molecular processes and cellular features. The therapeutic targets and mechanisms are also numerous and address diverse types of biology, such as angiogenesis, cognate immune regulation, tumor metabolism, hormonal regulation, and death pathways. With this 
diversity in mind, it raises the possibility that non-invasive MIBA that targets well-chosen biomarkers of cancer could improve early diagnosis and prediction of treatment efficacy. A variety of NIR fluorescent molecular imaging probes are available, and/or are reported, for powerful biological characterization of tumor biology, including probes for $\mathrm{pH}$ $[16,17]$, hypoxia [18, 19], protease activity [20-22], vascular leak [23, 24], integrin expression [21, 25], metabolic markers [26, 27], hormone receptors [28, 29], and well-characterized tumor markers [30-32]. However, their utilities as tools for MI vs. MIBA can be as much dependent on proper study design as on probe performance, with controls, treatment design, and imaging time points dictating whether results are geared toward just overt outcomes or toward gathering useful biological information. For example, in a recent publication [27], we illustrated the concept of MIBA-2 with two metabolic probes, BombesinRSense $^{\mathrm{TM}} 680$ and Transferrin-Vivo ${ }^{\mathrm{TM}} 750$, which were used to characterize treatment efficacy of the multikinase inhibitor sorafenib on HCT-116 tumors. These are probes that detect tumor expression of bombesin and transferrin receptors, associated with autocrine growth and iron uptake, respectively. When these probes were used as terminal readouts for treatment (MI-6), the results correlated well with tumor volume and bioluminescence and provided little information regarding tumor biology, other than proving that smaller, regressing tumors show proportionally less fluorescent signal (Fig. 2, left panel). However, earlier time points (at 2 or 3 days post-treatment) revealed the capability of these fluorescent probes to detect significant, disproportionate metabolic changes in the tumors prior to any overt changes in tumor volume or bioluminescence in a manner that agreed well with $\left[{ }^{18} \mathrm{~F}\right] \mathrm{FDG} / \mathrm{PET}$ imaging of decreased glucose uptake/metabolism at early times posttreatment (Fig. 2, right panel).

Probes specific for tumor metabolism markers are not the only way to exploit MIBA to better understand the biological impact of cancer treatment. Ma, et al. [33], illustrated the MIBA-2 strategy by using the $\alpha \mathrm{V} \beta 3$ integrin-specific NIR probe, IntegriSense ${ }^{\mathrm{TM}} 680$, to detect and quantify treatment efficacy with cisplatin/bevacizumab or refametinib 1 week prior to tumor changes seen by $\mu \mathrm{CT}$, allowing clear interpretation of biological effects independent of gross size changes. Ackerman, et al. [23], used a vascular NIR FL probe, AngioSense ${ }^{\circledR} 680$, to establish that the maximal anti-vascular effect of a potent antiangiogenic drug, CT-322, occurred within $24 \mathrm{~h}$ after the first drug administration, and this response mechanistically preceded the inhibition of tumor progression. The measurement of functional vascular leakiness with a vascular probe provided data that could not be easily achieved with histology or vascular corrosion casting. Other researchers have had similar findings, with Zhang and colleagues, in 2011 [34], using early time point imaging of AngioSense 750 to show that anti-VEGF treatment normalizes vascularity and decreases vascular leak. In a well-designed MIBA-2 study, Gao, et al. [35] established that A549-luc tumor size, bioluminescence, and $\left[{ }^{18} \mathrm{~F}\right] \mathrm{FDG}$ uptake were unaffected
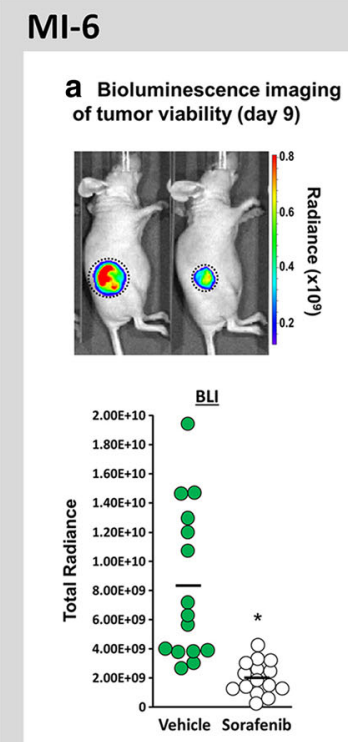
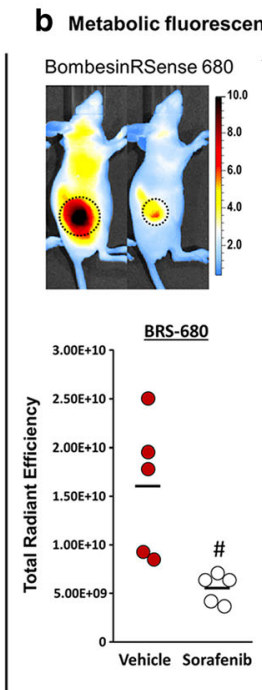

\section{MIBA-2}
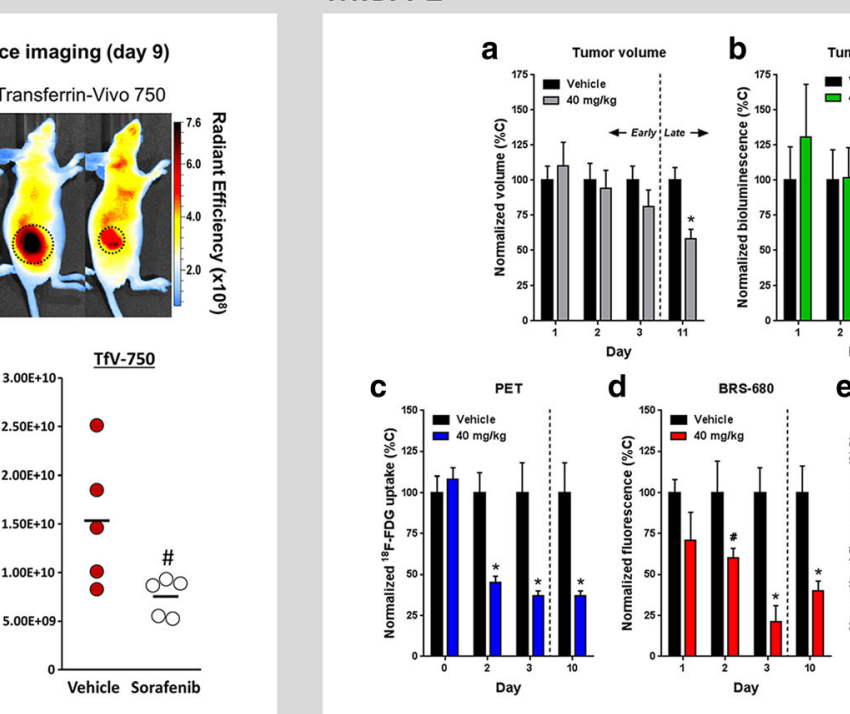

Fig. 2. Left panel: sorafenib treatment $(40 / \mathrm{mg} / \mathrm{kg} / \mathrm{d}$ PO for 5 days/week) was effective in decreasing HCT116-luc2 tumor growth as measured by a bioluminescence and b NIR fluorescence imaging with BombesinRSense 680 (BRS-680) or transferrin-vivo 750 (TfV-750) on day 9. Right panel: MIBA-2 imaging on day 2-3 using PET and optical imaging. a Tumor volume, $\mathbf{b}$ tumor bioluminescence, $\mathbf{c}\left[{ }^{18} \mathrm{~F}\right] \mathrm{FDG} / \mathrm{PET}$ imaging, d BRS-680 imaging, and e TfV-750 imaging. $\left(\# p<0.05,{ }^{*} p<0.01\right)$. This research was originally published in PLoS One by Tseng et al. [27]. These figures are unaltered and use is permitted under the Creative Commons License CC BY 4.0 (https://creativecommons.org/licenses/by/4.0/legalcode). 
after 7 days of dasatinib treatment (Fig. 3, left panel). However, molecular imaging strategies selected for detection of antiangiogenic effects, including VEGF detection by NIR fluorescent imaging of ranibizumab-DyLight755, showed decreased tumor uptake even though overt changes in tumors were not evident up to 25 days post-treatment (Fig. 3, right panel). Further corroboration of the antiangiogenic effects of dasatinib was established in an in vivo matrigel plug assay, showing clear dasatinib-induced antiangiogenic effects (i.e., reduced VEGF as indicated by decreased probe uptake) as compared with the vehicle group. Dasatinib also showed synergistic effects with docetaxel (not shown here); however, dasatinib effects alone would have been difficult to characterize in vivo without imaging.

Another useful MIBA strategy in cancer is to measure increased cell death in tumors as a result of chemotherapy treatment with cytotoxic agents. Cell death can be detected by designing probes that specifically, or by charge interaction, bind to phosphatidylserine (PS) molecules that translocate to the outer leaflet of cell membranes of cells undergoing apoptosis or early necrosis. This can be achieved with NIR fluorescent-labeled annexin V (which is a protein that interacts with PS), by labeled anti-PS antibodies, or by positively charged labeled molecules selected for PS binding. Gee, et al. [36] assessed cell death and Her-2 expression changes 3-4 days following trastuzumab injection and showed dramatic increases in SKBR-3 tumor accumulation of Annexin V Cy5.5, while simultaneously showing a decrease in tumor trastuzumab-AlexaFluor750 (Tras-AF750), attributed to receptor blockade. As controls, two Her-2-expressing, but resistant, control tumors showed trastuzumab-induced blockade of tumor Tras-AF750 but did not show increased tumor Annexin V Cy5.5. Thus, non-invasive MIBA was able to capture key biological factors in response to treatment efficacy/nonefficacy in agreement with in vitro characterizations. Similarly, a well-designed study by Manning, et al. [37], used NIR fluorescent-labeled EGF to assess EGFR blockade by cetuximab, while simultaneously showing increased cell death with NIR-labeled annexin V. These results were obtained early in treatment, prior to overt tumor regression and without changes in $3^{\prime}$-dexoy- $3^{\prime}-\left[{ }^{18} \mathrm{~F}\right]$ fluorothymidine/PET imaging. We have also established that a commercially available annexin $\mathrm{V}$ probe (Annexin Vivo ${ }^{\mathrm{TM}} 750$ ) can be used to assess drug-induced tumor killing by chemotherapy. A single dose of cyclophosphamide was given to HT-29 tumor-bearing nu/nu mice, Annexin Vivo 750 was injected intravenously $24 \mathrm{~h}$ later, and mice were imaged at $2 \mathrm{~h}$. This acute treatment had no effect on tumor size, but $30 \%$ of animals showed $3-9 \times$ higher tumor signal with the overall mean of all tumors increasing more than twofold [JD Peterson, unpublished data].

\section{MIBA Strategies in Inflammation}

Inflammation is an important aspect of many chronic diseases, including cancer, cardiovascular disease, arthritis, COPD/asthma, gastrointestinal disease, autoimmunity, demyelinating disease, and even obesity. Multiple structural and anatomical changes can occur during inflammation, including edema, fibrosis, and overt tissue destruction/ damage, that can be readily captured by various conventional imaging techniques (like microCT, MRI, and ultrasound). However, understanding and localizing early inflammatory foci and biological processes that precede substantial anatomical changes are also important. Nuclear imaging has generally become the established tool for MI of

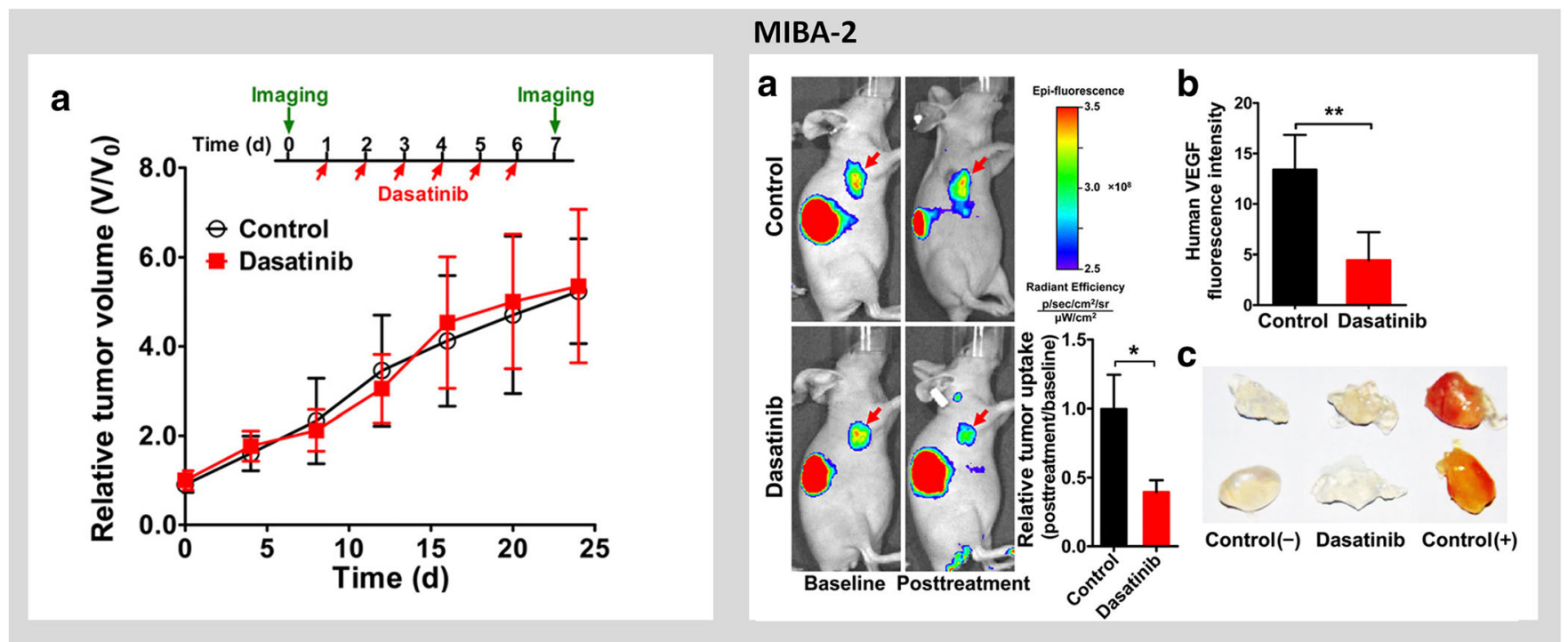

Fig. 3. Left panel: dasatinib treatment ( $40 / \mathrm{mg} / \mathrm{kg} / \mathrm{d}$ for 6 days) ineffective in altering A549-fLuc tumor growth as measured by tumor volume. Right panel: a VEGF detection using dye755-ran shows post-dasatinib inhibition of VEGF expression despite lack of treatment effect on tumor volume. b Ex vivo imaging confirms decrease in tumor VEGF levels. c Inhibition of growth factor-driven angiogenesis by dasatinib in matrigel plug assay. $\left({ }^{*} p<0.05,{ }^{\star *} p<0.01\right)$. This research was originally published in the Journal of Nuclear Medicine by Gao et al. [35] () by the Society of Nuclear Medicine and Molecular Imaging, Inc. 
inflammatory conditions, for example, using Ga-67, $\left[{ }^{18} \mathrm{~F}\right] \mathrm{FDG}$, Tc-99-m-labeled antibodies, and leukocytes labeled with $\mathrm{Cu}-64, \mathrm{Ga}-68$ or I-124. However, there are also a number of NIR fluorescent MI probes for detecting changes in vascularity, protease activity, metabolism, specific biomarker expression, and different types of tissue damage. In addition, there are NIR fluorescent cell labeling agents that allow short-term tracking of multiple leukocyte populations. The ease and cost-effectiveness of non-invasive fluorescence MI techniques make this an important tool for earlier and more reliable prognosis, earlier assessment of treatment response, and the development of new therapeutics.

Some publications in fluorescence inflammation imaging particularly stand out for their use of non-invasive fluorescence MI in challenging animal models and for their significant corroborating biology validation. For example, researchers have made extensive progress in using fluorescence tomography for detecting very early, non-invasive detection of cardiovascular disease by measuring macrophage protease activity, nanoparticle uptake, and/or integrin expression [38-42]. Pulmonary inflammation also presents challenges for non-invasive MI, due to imaging depth, heart interference, and complications of fluorescence imaging of air-filled tissue. Some of the research involves straightforward biological endpoint MIBA-3 approaches that use protease-activatable probes to detect pulmonary fibrosis [43], asthma [44, 45], and LPS-induced pulmonary inflammation $[46,47]$ that highlight the advantages of imaging and its correlation to inflammatory cell counts. Stellari, et al. [48], used a MIBA-1, 3 strategy with ProSense ${ }^{\circledR} 680$ and MMPSense ${ }^{\circledR} 680$ probes (for cathepsin and MMP activity, respectively) to assess both inflammation and fibrosis in dust mite, ragweed, and aspergillus (DRA)-induced asthma and airway remodeling (Fig. 4). The imaging results they obtained correlated well with ex vivo readouts, such as bronchoalveolar lavage (BAL) cell analysis, inflammatory cell infiltration, and histological analysis of airway epithelium hyperplasia and collagen deposition, thus showing the ability to non-invasively profile biological changes in inflammation and tissue remodeling in disease progression and treatment. Typically, ProSense and MMPsense cannot discriminate between inflammation and fibrosis; however, the clever assessment of early ProSense (at the $\mathrm{d} 8$ of peak inflammation) and late MMPSense (d11 low inflammation, decreased ProSense, but retained/increasing MMPSense signal, suggesting fibrosis) allowed the authors to separately interpret relative levels of inflammation and fibrosis. Their research paves the way for two-channel cathepsin/MMP activity ratios that can discriminate non-invasively between inflammatory stages of DRA-induced asthma from fibrotic stages; for example, their data suggests a fivefold difference in MMP:cathepsin activity ratios when comparing the peak of inflammation (d8) to the onset of fibrosis (d11).

In mouse arthritis imaging, several publications either show detection of early disease $[49,50]$ or superior treatment efficacy readouts [51-54] classifying these as MIBA-1 and MIBA-3 approaches, respectively. Other researchers have provided excellent and highly validated fluorescence MI results correlating with clinical and histological outcomes $[55,56]$ but did not necessarily support the MI readouts as more valuable than established readouts like paw swelling and clinical scoring. At times, the difference between MI and MIBA is in the study design; MIBA strategies generate biological interpretation, which can often require an appropriate study design or the right treatment groups to fully test the in vivo model. For example, in 2017 [52], we compared a COX-2 inhibitor (Celebrex), a p38 MAPK inhibitor (SD0006), and prednisolone treatment in collagen antibody-induced arthritis (CAIA) and imaged with two probes to detect inflammation (ProSense 750, MMPSense 680) and one to detect bone turnover (OsteoSense ${ }^{\circledR}$ 680). Thus, this study was designed to compare a non-disease-modifying anti-rheumatic drug (non-DMARD), using Celebrex, to a DMARD treatment that inhibits the TNF pathway (SD0006). Prednisolone ablated the disease and served primarily to show maximal treatment effect. The two non-steroidal treatments yielded similar effects on overt paw swelling, but only the DMARD treatment affected the underlying inflammatory destruction. Imaging with ProSense 750 and MMPSense 680 clearly showed that Celebrex, despite inhibition of paw swelling had no effect on underlying disease, whereas SD0006 inhibited both swelling and underlying disease (Fig. 5, left panel). Current standard arthritis metrics, paw swelling and clinical score, correlated poorly with histology inflammation scores; however, imaging results with the two proteaseactivatable probes correlated very well with the underlying histological inflammation (Fig. 5, right panel). OsteoSense (not shown here) corroborated the decrease in bone destruction, only with DMARD treatment, and even detected late-stage prednisolone-induced osteoporosis in mice cured of arthritis by steroid treatment (Peterson 2010). Thus, this MIBA-3 approach yielded considerable biological data, including the ability to non-invasively discriminate between DMARD and non-DMARD treatment and to detect steroidinduced side effects on bone turnover.

A study by Scales et al. [56] used a very similar study approach in collagen-induced arthritis (CIA) but with only steroid treatment (dexamethasone) and using MMPSense 750 FASTTM, ProSense 750 FAST, Neutrophil Elastase 680 FAST to assess inflammation, and Cat K 680 FAST to assess bone resorption activity. Imaging was performed at the time of maximal steroid inhibition of disease, and all readouts (including paw swelling, clinical score, and imaging) showed excellent inhibition of signal by treatment. All imaging readouts correlated with paw swelling, clinical scores, and histopathology severity, which is a great validation of probe performance, yet this study appeared to be intentionally designed as MI-6 and not designed as MIBA. Rather, the authors appeared to focus on validation of imaging readouts to conventional metrics rather than 


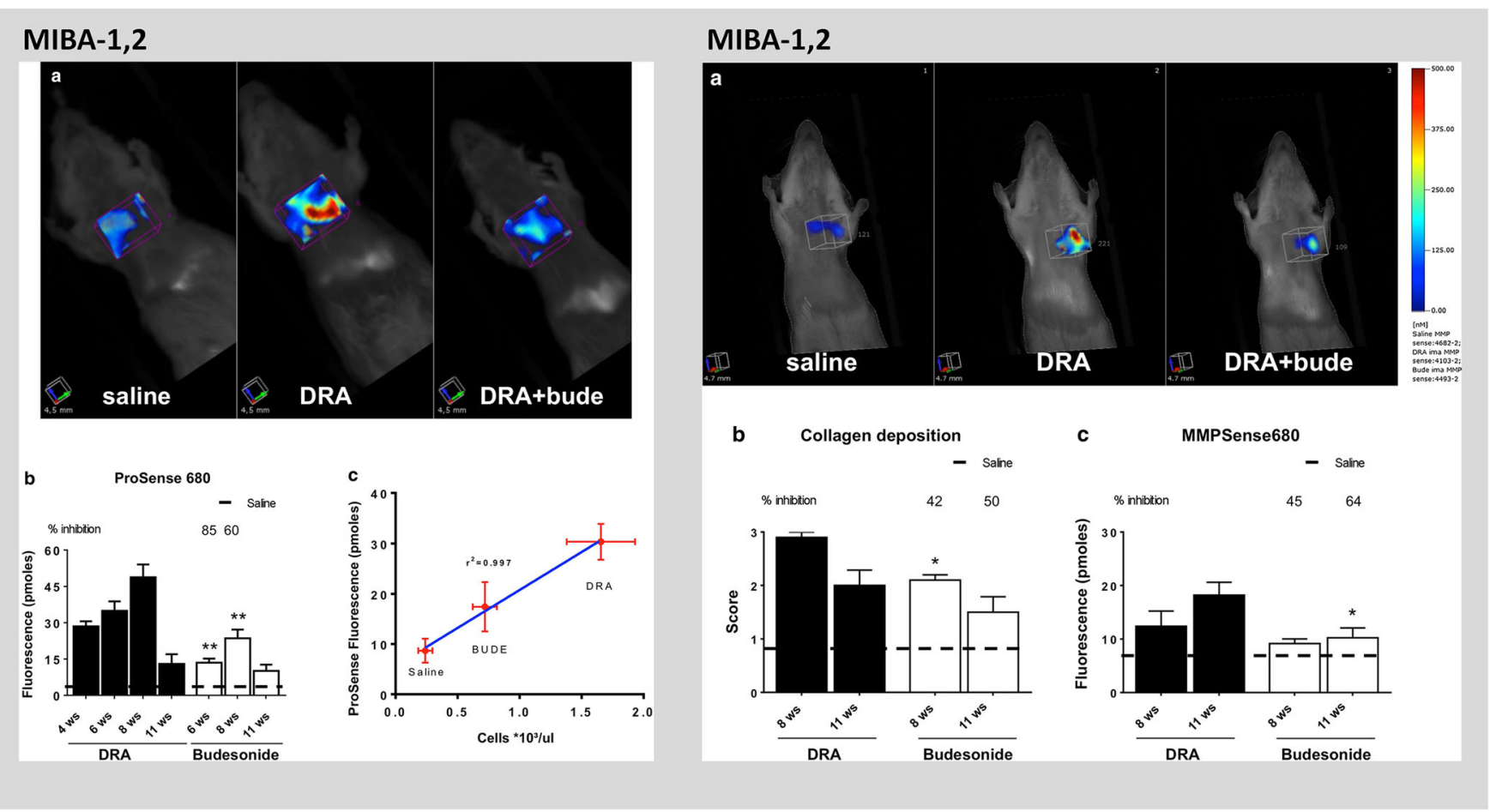

Fig. 4. Left panel: ProSense 680 imaging of DRA-induced pulmonary inflammation and inhibition by budesonide. aln vivo imaging by FMT 2500 at 8 weeks. b Fluorescence tomographic quantification at 4-11 weeks compared to budesonide treatment. c Correlation between ProSense 680 imaging and BAL cell counts. Right panel: MMPSense 680 imaging of DRAinduced pulmonary fibrosis and budesonide efficacy. aln vivo imaging by FMT 2500 at 11 weeks. b Masson's trichrome staining of formalin-fixed, paraffin embedded lung tissue sections for collagen deposition and sections were semi-quantitatively scored at 8 and 11 weeks. c Fluorescence tomographic quantification at 8 and 11 weeks compared to budesonide treatment. $\left({ }^{\star} p<0.05,{ }^{* *} p<0.01\right)$. This research was originally published in the Journal of Translational Medicine by Stellari et al. [48]. These figures are unaltered and use are permitted under the Creative Commons License CC BY 4.0 (https://creativecommons.org/ licenses/by/4.0/legalcode).

MIBA-3

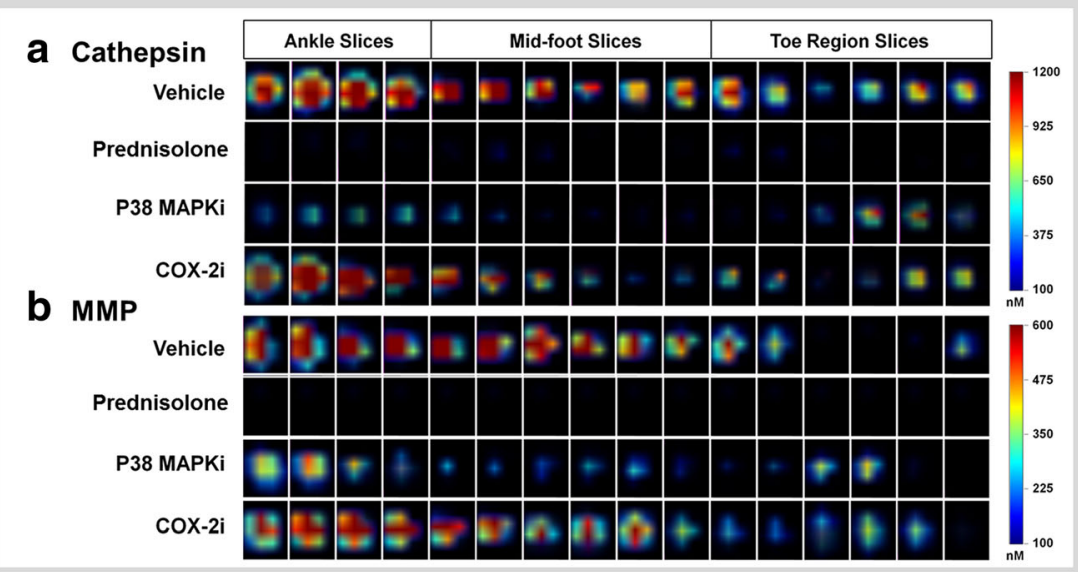

\section{MIBA-3}

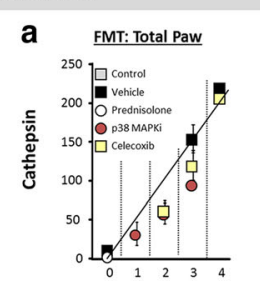

b

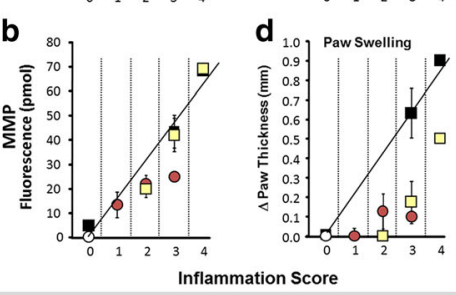

Fig. 5. Left panel: axial tomographic slices of non-invasive hind paw imaging datasets using a ProSense 750 (cathepsin) and $\mathbf{b}$ MMPSense 680 (MMP) acquired in untreated, treated, and vehicle mice. Images are axial slices of tomographic fluorescence from individual mice that are representative of the mean of each experimental group. Right panel: correlation of paw imaging a ProSense 750 (cathepsin), b MMPSense 680 (MMP), c clinical score, and d paw swelling data with histology inflammation scoring. This research was originally published in Arthritis Research and Therapy by Peterson, et al. [52]. These figures are unaltered and are permittedly used under the Creative Commons License CC BY 4.0 (https://creativecommons.org/licenses/ by/4.0/legalcode). 
using imaging itself to prove the added value provided by imaging probes.

\section{MIBA Strategies in Toxicology/Safety}

Drug-induced liver and kidney injury can be reasons for termination of drug discovery research projects, failure of novel drugs in late-stage clinical trials, and FDA withdrawal of drugs from the commercial market. The later the discovery of liver or kidney toxicity findings are made, the higher the costs incurred, which increases the overall cost of pharmaceutical R\&D and the ultimate drug-pricing for patients. This means that early identification of drug candidates that have the potential for adverse effects on kidney or liver function is an important priority in the drug discovery and development process for both safety and financial reasons. NIR fluorescence MI offers a useful approach to non-invasively assess drug effects in preclinical testing, and there are opportunities for MIBA strategies, in particular, that may help to understand in situ dynamic biological changes in tissue. There are a number of published or commercial probes with the potential to detect relevant toxicological processes, including tissue perfusion kinetics (NIR dyes, ICG), kidney glomerular filtration kinetics (NIR FLlabeled inulin), cell death (annexin V probe), caspase activity (activatable probes), inflammation (cathepsin-activatable, MMP-activatable, elastase-activatable, folate-targeted probes), fibrosis (MMP-activatable, peptidomimetic probes), reactive oxygen/nitrogen species (ROS/RNS probes), and others. It is important, however, to express the significant caveat that MI and MIBA approaches (particularly if performed in mice) are more likely to be of use in very early screening/triaging of compounds early in the drug discovery process rather than in the late stages of development and formal toxicology. Nevertheless, such an early MI strategy could serve to focus R\&D activities on chemical compounds with less potential for showing adverse effects in later stage development.

Non-invasive fluorescence imaging of drug-induced tissue injury is a very new area of interest, so there is really very little published research at this time. However, publications in drug-induced liver and kidney injury, as well as non-drug-induced models of liver/kidney injury, support the potential for MIBA approaches in toxicology testing in vivo. First, there are a couple of reports of the use of simple MI-7 approaches that exploit physiologic perfusion characteristics of NIR fluorescent dyes. Abulrob et al. [57] showed that fluorescence intensity and lifetime of intravenously injected Cy5.5 were clearly reduced in ischemic kidneys, suggesting significant effects on kidney perfusion/ clearance kinetics following acute ischemic damage. The results aligned with histologic observations of morphology changes in ischemic kidneys, including tubular dilatation, flattened tubular epithelium, and luminal debris. Tubular epithelial cells displayed nuclear changes indicative of apoptosis and necrosis. In 2012, Amoozegar et al. [58] examined MIBA- 4 changes in tissue, induced with carbon tetrachloride $\left(\mathrm{CCl}_{4}\right)$ dosing, by measuring the altered dynamics of indocyanine green (ICG) perfusion and accumulation in tissues. The flow and clearance dynamics of ICG in several organs/tissues, including kidney and liver, were assessed by epifluorescence dynamic contrast enhanced (DyCE) imaging before and after $\mathrm{CCl}_{4}$ administration. ICG liver uptake was delayed, and kidney uptake was accelerated by $\mathrm{CCl}_{4}$-induced injury. Two days later, tissue uptake in tissues returned to near normal rates. Results were in agreement with elevations in blood alanine aminotransferase (ALT), indicating liver damage, and ICG results were attributed to decreases in liver uptake/processing of albuminbound ICG and perfusion changes in kidneys. A targeted probe has also been used effectively to assess tissue changes in kidney nephritis models. Nakamura and Tabata [59] used three different mouse models of nephritis and imaged with a Cy7-labeled integrin-targeted probe. The probe accumulated at greater levels in inflamed kidneys, as compared to normal kidneys, $24 \mathrm{~h}$ after administration, and the renal pattern of labeling suggested that the probe was non-invasively detecting infiltrating macrophages. These three studies provide useful non-invasive imaging techniques, essentially providing surrogate markers that align with other metrics, such as blood markers and histology. Pushing the limits of these assessments to show changes earlier or at lesser severity may reveal the early and subtle perfusion or cellular influx changes that precede overt tissue damage.

One of the best examples of MIBA-4 in drug-induced liver injury was published by Shuhendler, et al. [14]. They used a combined fluorescent/chemiluminescent probe $(\mathrm{CF}$ SPN 775) designed to detect reactive nitrogen species (RNS) and reactive oxygen species (ROS), and non-invasively detected nitrosative/oxidative stress in the liver associated with acetaminophen (APAP)-induced toxicity in female nu/ nu mice. $C F$-SPN 775 was able to detect ROS/RNS changes in the liver within 20-60 min using non-invasive epifluorescence and chemiluminescence imaging on the IVIS spectrum. Although liver signal increases were only seen at the very highest dose of $300 \mathrm{mg} / \mathrm{kg}$, these increases occurred at least $2 \mathrm{~h}$ prior to overt histological signs of liver damage. A broader MIBA-4 approach to drug-induced liver injury was taken by us in 2017 [15] in which a cocktail (AMT-750) of three NIR fluorescent imaging probes was used that measured apoptosis/early necrosis, inflammation/ fibrosis, and iron metabolism (Annexin Vivo 750, MMPSense 750, and Transferrin-Vivo 750, respectively). A cocktail of probes, all at $750 \mathrm{~nm}$ excitation, rather than individual probes at different wavelengths, was used to simplify the concept of very early discovery compound screening approach in the belief that the screening itself (i.e., flagging potential toxicity) was more important than profiling specific biological changes with toxicity. This strategy allowed the effective detection of different types of liver injury, including hepatocellular, cholestatic, and combined hepatocellular/cholestatic induced by four different chemicals/drugs (Fig. 6). Comparison to vascular probe 
uptake allowed both the simultaneous detection of vascular damage as well as correction for potential non-specific uptake of the targeted/activatable probes (not shown). Biological changes detected by MIBA were generally seen prior to overt histologic damage, and for some drugs, even the in vivo imaging response was reversible within $24 \mathrm{~h}$. Interestingly, the measured drug-induced biological changes, imaged with AMT-750, were not restricted to the liver, detecting changes in a variety of tissues, including kidneys, spleen, stomach, and adipose tissue. Most, if not all, of these tissue changes correlated with published findings of tissue toxicities that were observed upon longer-term drug dosing. Early detection was shown in the publication, but it was also evident in other unpublished studies that hepatocellular tox could also be seen at lower single bolus doses with APAP (as low as $200 \mathrm{mg} / \mathrm{kg}$ ) and TAA (as low as $100 \mathrm{mg} / \mathrm{kg}$ ) (Peterson, unpublished observations).

Another in vivo liver injury study worthy of note as a MIBA-4 strategy was published in 2012 by Ozaki et al. [60].
An adenovirus vector encoding caspase-3 probe (AdpcFlucDEVD) was injected intravenously 3 days prior to induction of experimental liver ischemia/reperfusion injury. Luciferin substrate was injected at different times relative to ischemia/ reperfusion to generate a bioluminescent signal proportional to the upregulation of caspase-3 activity (indicating apoptosis) (Fig. 7). Bioluminescence signal was optimal with a 60min ischemic event, and imaging was optimal $6 \mathrm{~h}$ after ischemia. Interestingly, only the comparison of in vivo imaging data with serum glutamic pyruvic transaminase (GPT) and lactate dehydrogenase (LDH) results allowed the distinction between $6 \mathrm{~h}$ apoptosis and $9 \mathrm{~h}$ necrosis. This approach may be mostly restricted to the liver due to practical limitations of adenovirus use; however, the potential of using different reporter constructs, or perhaps a set of appropriate transgenic mice, could make this a very sensitive and informative method for early screening/characterization of various biological changes associated with different types of drug-induced liver injury.

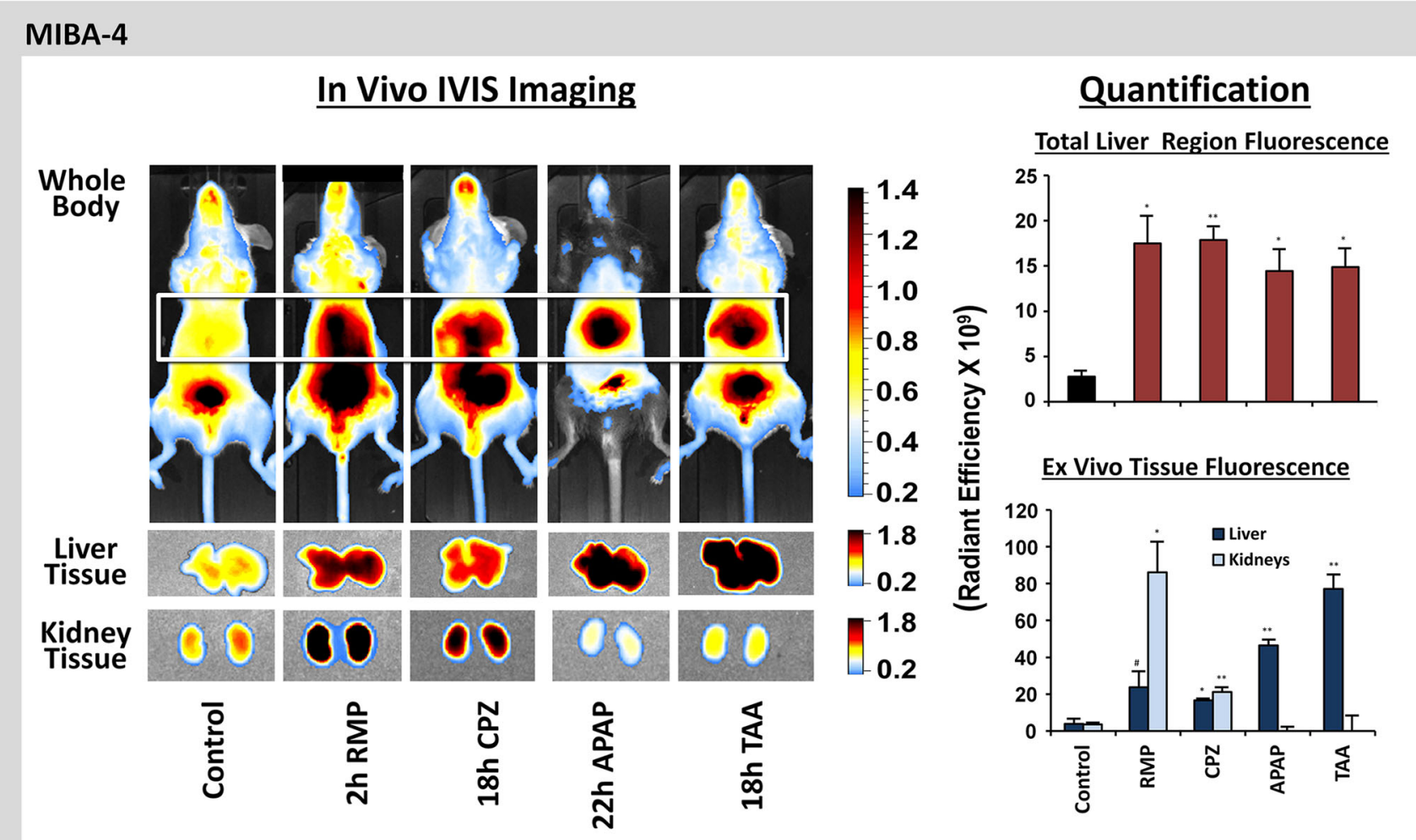

Fig. 6. Validation of a three-probe fluorescent cocktail for general detection of drug-induced tissue injury. Drug-dosed mice were injected with the imaging cocktail (AMT-750) at two different times post-treatment (18 h for TAA and APAP at $300 \mathrm{mg} / \mathrm{kg}$; $2 \mathrm{~h}$ for CPZ [100 mg/kg] and RMP [300 mg/kg]), and all mice were imaged $24 \mathrm{~h}$ after AMT-750 for both non-invasive liver assessment (upper left panel) and ex vivo tissues (lower left panel). Quantification of the liver signal from non-invasive imaging (upper right panel) and the ex vivo liver and kidney signal (lower right panel) were determined by region-of-interest placement to capture the entire liver or individual tissues, and results were represented as the total fluorescent signal. Statistical significance was assessed by analysis of variance with the Dunnett post-test $\left({ }^{*} p<0.01 ;{ }^{* *} p<0.001 ; \# p<0.05\right)$. This research was originally published in the Journal of Pharmacology and Experimental Therapeutics by Vasquez and Peterson [15]. This figure is unaltered and use is permitted under the Creative Commons License CC BY 4.0 (https://creativecommons.org/licenses/by/4.0/ legalcode). 


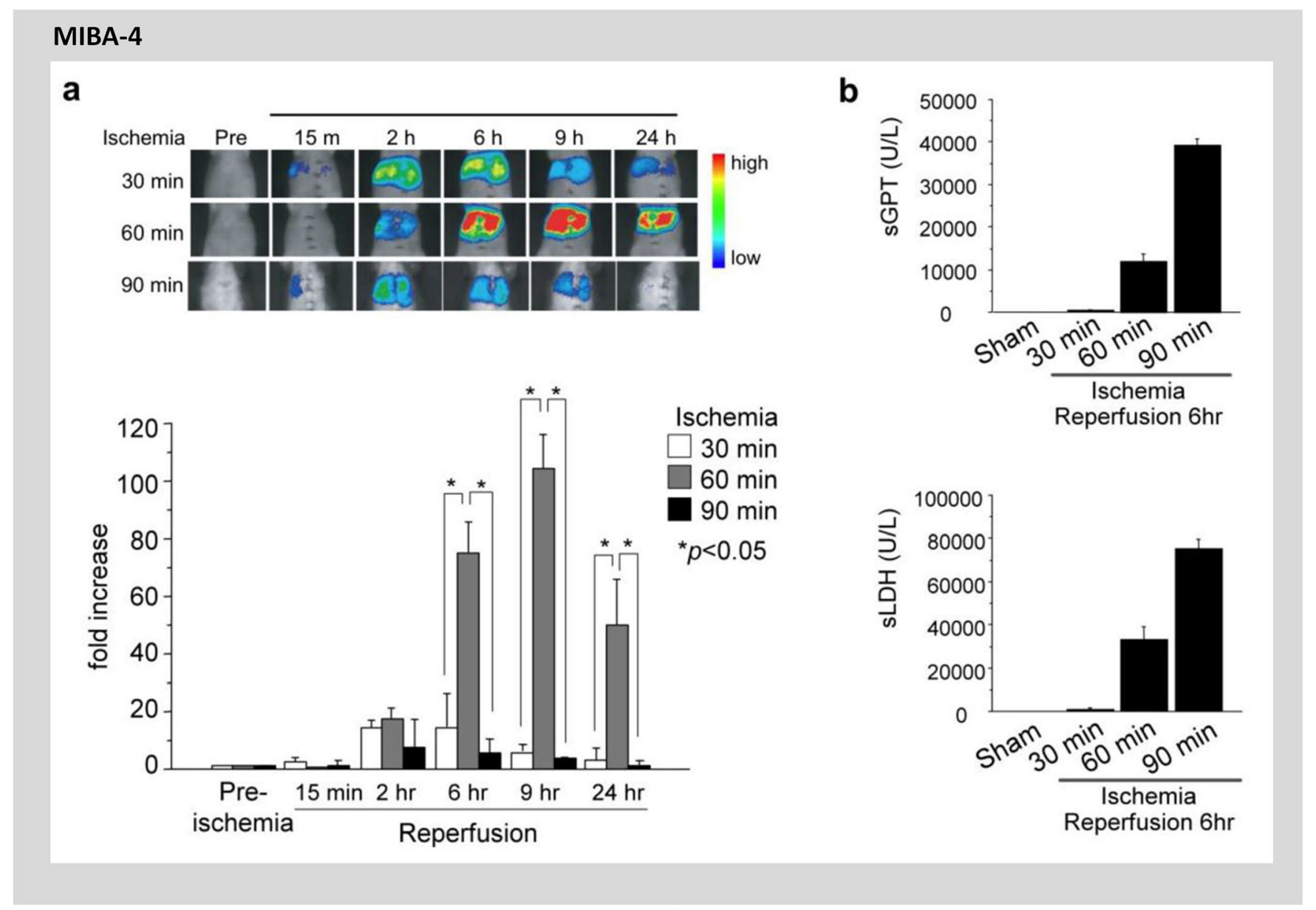

Fig. 7. In vivo use of adenovirus vector encoding caspase-3 bioluminescence probe (AdpcFluc-DEVD) to image ischemiainduced liver damage. a Ischemic insult to liver induced caspase-3 activation at 15 min, 2, 6, 9, and 24 h. b Serum levels of GPT and LDH (necrotic markers of liver) $6 \mathrm{~h}$ after hepatic ischemia/reperfusion. This research was originally published in Theranostics by Ozaki et al. [60]. This figure is unaltered and use is permitted under the Creative Commons License CC BY-NCSA 3.0 (https://creativecommons.org/licenses/by-nc-sa/3.0).

\section{Conclusion}

Advances continue to be made in fluorescence MI and the development of novel NIR fluorescent imaging probes specific for important biomarkers, and these advances are allowing MI to move out of predominant cancer imaging and into a variety of other diseases and models. This review covered concepts and examples to guide researchers in their considerations about biomarkers and how to design effective studies that maximize the acquisition of useful biological information from living animals, i.e., the application of MIBA approaches. Too often, well-designed and well-validated fluorescent probes are utilized in a manner that does not add biological information beyond the knowledge that a given probe of a known specificity can detect disease or therapeutic efficacy. It is also important to focus not only on best practices but also to bear in mind the practicalities of MI; the extra expense per mouse of the imaging probes must always be warranted and not just provide a novel and more expensive way to achieve the same interpretation as cheaper, non-imaging readouts.

The application of fluorescence MI, MIBA in particular, to important in vivo biological questions will continue to grow as long as researchers continue to push the envelope with regard to development, characterization, and application of novel fluorescent imaging. In return, MI offers valuable research tools that in many cases can serve to reduce and refine animal usage, often generating more information from fewer animals or from the same number of animals. Moving into other therapeutic areas, beyond the preponderance of cancer research, and deeper into application areas like autoimmunity, obesity, diabetes, cardiovascular disease, infectious disease, and toxicology will further spread the benefits of fluorescence MIBA.

Acknowledgments. I would like to acknowledge Kristine Vasquez and JenChieh Tseng for providing critical studies and study designs in support of ongoing bio-imaging, some of which is cited in this review.

Funding Sources. No funding was required for the generation of this review article, but the author's salary was paid by his employer, PerkinElmer, Inc.

Compliance with Ethical Standards

\section{Conflict of Interest}

The author declares that he has no conflict of interest. 
Open Access This article is distributed under the terms of the Creative Commons Attribution 4.0 International License (http:// creativecommons.org/licenses/by/4.0/), which permits unrestricted use, distribution, and reproduction in any medium, provided you give appropriate credit to the original author(s) and the source, provide a link to the Creative Commons license, and indicate if changes were made.

\section{References}

1. Alavi A, Dann R, Chawluk J, Alavi J, Kushner M, Reivich M (1986) Positron emission tomography imaging of regional cerebral glucose metabolism. Semin Nucl Med 16:2-34

2. Haberkorn U, Markert A, Mier W, Askoxylakis V, Altmann A (2011) Molecular imaging of tumor metabolism and apoptosis. Oncogene 30:4141-4151

3. Rice L, Bisdas S (2017) The diagnostic value of FDG and amyloid PET in Alzheimer's disease-a systematic review. Eur J Radiol 94:16-24

4. McMahon MT, Chan KW (2014) Developing MR probes for molecular imaging. Adv Cancer Res 124:297-327

5. Sosnovik DE, Weissleder R (2007) Emerging concepts in molecular MRI. Curr Opin Biotechnol 18:4-10

6. Voigt JU (2009) Ultrasound molecular imaging. Methods 48:92-97

7. Ashton JR, West JL, Badea CT (2015) In vivo small animal micro-CT using nanoparticle contrast agents. Front Pharmacol 6:256

8. Deliolanis N, Lasser T, Hyde D, Soubret A, Ripoll J, Ntziachristos V (2007) Free-space fluorescence molecular tomography utilizing 360 degrees geometry projections. Opt Lett 32:382-384

9. Mohajerani P, Adibi A, Kempner J, Yared W (2009) Compensation of optical heterogeneity-induced artifacts in fluorescence molecular tomography: theory and in vivo validation. J Biomed Opt 14:034021

10. Schulz RB, Ripoll J, Ntziachristos V (2003) Noncontact optical tomography of turbid media. Opt Lett 28:1701-1703

11. Schulz RB, Ripoll J, Ntziachristos V (2004) Experimental fluorescence tomography of tissues with noncontact measurements. IEEE Trans Med Imaging 23:492-500

12. Mankoff DA (2007) A definition of molecular imaging. J Nucl Med $48(18 \mathrm{~N}): 21 \mathrm{~N}$

13. Peterson JD (2016) Noninvasive in vivo optical imaging models for safety and toxicity testing. In: Gupta RC (ed) Nutraceuticals - efficacy, safety and toxicity. Elsevier, pp 306-317

14. Shuhendler AJ, Pu K, Cui L, Uetrecht JP, Rao J (2014) Real-time imaging of oxidative and nitrosative stress in the liver of live animals for drug-toxicity testing. Nat Biotechnol 32:373-380

15. Vasquez KO, Peterson JD (2017) Early detection of acute druginduced liver injury in mice by noninvasive near-infrared fluorescence imaging. J Pharmacol Exp Ther 361:87-98

16. Gilson RC, Tang R, Som A, Klajer C, Sarder P, Sudlow GP, Akers WJ, Achilefu S (2015) Protonation and trapping of a small pH-sensitive nearinfrared fluorescent molecule in the acidic tumor environment delineate diverse tumors in vivo. Mol Pharm 12:4237-4246

17. Ma J, Li W, Li J, Shi R, Yin G, Wang R (2018) A small molecular $\mathrm{pH}$-dependent fluorescent probe for cancer cell imaging in living cell. Talanta 182:464-469

18. Bao B, Groves K, Zhang J, Handy E, Kennedy P, Cuneo G, Supuran CT, Yared W, Rajopadhye M, Peterson JD (2012) In vivo imaging and quantification of carbonic anhydrase IX expression as an endogenous biomarker of tumor hypoxia. PLoS One 7:e50860

19. van Brussel AS, Adams A, Oliveira S et al (2016) Hypoxia-targeting fluorescent nanobodies for optical molecular imaging of pre-invasive breast cancer. Mol Imaging Biol 18:535-544

20. Bremer C, Tung CH, Bogdanov A Jr, Weissleder R (2002) Imaging of differential protease expression in breast cancers for detection of aggressive tumor phenotypes. Radiology 222:814-818

21. Kossodo S, Pickarski M, Lin SA, Gleason A, Gaspar R, Buono C, Ho G, Blusztajn A, Cuneo G, Zhang J, Jensen J, Hargreaves R, Coleman P, Hartman G, Rajopadhye M, Duong LT, Sur C, Yared W, Peterson J, Bednar B (2010) Dual in vivo quantification of integrin-targeted and protease-activated agents in cancer using fluorescence molecular tomography (FMT). Mol Imaging Biol 12:488-499

22. Mahmood U, Weissleder R (2003) Near-infrared optical imaging of proteases in cancer. Mol Cancer Ther 2:489-496
23. Ackermann M, Carvajal IM, Morse BA et al (2011) Adnectin CT-322 inhibits tumor growth and affects microvascular architecture and function in Colo205 tumor xenografts. Int J Oncol 38:71-80

24. Montet X, Figueiredo JL, Alencar H, Ntziachristos V, Mahmood U, Weissleder R (2007) Tomographic fluorescence imaging of tumor vascular volume in mice. Radiology 242:751-758

25. Hensley HH, Roder NA, O'Brien SW, Bickel LE, Xiao F, Litwin S, Connolly DC (2012) Combined in vivo molecular and anatomic imaging for detection of ovarian carcinoma-associated protease activity and integrin expression in mice. Neoplasia 14:451-462

26. Lee H, Kim J, Kim H, Kim Y, Choi Y (2014) A folate receptorspecific activatable probe for near-infrared fluorescence imaging of ovarian cancer. Chem Commun (Camb) 50:7507-7510

27. Tseng JC, Narayanan N, Ho G, Groves K, Delaney J, Bao B, Zhang J, Morin J, Kossodo S, Rajopadhye M, Peterson JD (2017) Fluorescence imaging of bombesin and transferrin receptor expression is comparable to ${ }^{18} \mathrm{~F}$-FDG PET in early detection of sorafenib-induced changes in tumor metabolism. PLoS One 12:e0182689

28. Hu Z, Yang L, Ning W, Tang C, Meng Q, Zheng J, Dong C, Zhou HB (2018) A high-affinity subtype-selective fluorescent probe for estrogen receptor beta imaging in living cells. Chem Commun (Camb) 54:3887-3890

29. Lee CW, Guo L, Matei D, Stantz K (2015) Development of folliclestimulating hormone receptor binding probes to image ovarian xenografts. J Biotechnol Biomater 5(3):198

30. Ding S, Blue RE, Moorefield E et al (2017) Ex vivo and in vivo noninvasive imaging of epidermal growth factor receptor inhibition on colon tumorigenesis using activatable near-infrared fluorescent probes. Mol Imaging 16:1536012117729044

31. Lwin TM, Murakami T, Miyake K, Yazaki PJ, Shivley JE, Hoffman RM, Bouvet M (2018) Tumor-specific labeling of pancreatic cancer using a humanized anti-CEA antibody conjugated to a near-infrared fluorophore. Ann Surg Oncol 25:1079-1085

32. Paudyal B, Paudyal P, Shah D, Tominaga H, Tsushima Y, Endo K (2014) Detection of vascular endothelial growth factor in colon cancer xenografts using bevacizumab based near infrared fluorophore conjugate. J Biomed Sci 21:35

33. Ma X, Phi Van V, Kimm MA et al (2017) Integrin-targeted hybrid fluorescence molecular tomography/X-ray computed tomography for imaging tumor progression and early response in non-small cell lung cancer. Neoplasia 19:8-16

34. Zhang Q, Bindokas V, Shen J, Fan H, Hoffman RM, Xing HR (2011) Time-course imaging of therapeutic functional tumor vascular normalization by antiangiogenic agents. Mol Cancer Ther 10:1173-1184

35. Gao L, Liu H, Sun X, Gao D, Zhang C, Jia B, Zhu Z, Wang F, Liu Z (2016) Molecular imaging of post-Src inhibition tumor signatures for guiding dasatinib combination therapy. J Nucl Med 57:321-326

36. Gee MS, Upadhyay R, Bergquist H, Weissleder R, Josephson L, Mahmood U (2007) Multiparameter noninvasive assessment of treatment susceptibility, drug target inhibition and tumor response guides cancer treatment. Int J Cancer 121:2492-2500

37. Manning HC, Merchant NB, Foutch AC, Virostko JM, Wyatt SK, Shah C, McKinley ET, Xie J, Mutic NJ, Washington MK, LaFleur B, Tantawy MN, Peterson TE, Ansari MS, Baldwin RM, Rothenberg ML, Bornhop DJ, Gore JC, Coffey RJ (2008) Molecular imaging of therapeutic response to epidermal growth factor receptor blockade in colorectal cancer. Clin Cancer Res 14:7413-7422

38. Glinzer A, Ma X, Prakash J, Kimm MA, Lohöfer F, Kosanke K, Pelisek J, Thon MP, Vorlova S, Heinze KG, Eckstein HH, Gee MW, Ntziachristos V, Zernecke A, Wildgruber M (2017) Targeting elastase for molecular imaging of early atherosclerotic lesions. Arterioscler Thromb Vasc Biol 37:525-533

39. Abd-Elrahman I, Kosuge H, Wises Sadan T, Ben-Nun Y, Meir K, Rubinstein C, Bogyo M, McConnell MV, Blum G (2016) Cathepsin activity-based probes and inhibitor for preclinical atherosclerosis imaging and macrophage depletion. PLoS One 11:e0160522

40. Lin SA, Patel M, Suresch D et al (2012) Quantitative longitudinal imaging of vascular inflammation and treatment by ezetimibe in apoE mice by FMT using new optical imaging biomarkers of cathepsin activity and alpha(v)beta(3) integrin. Int J Mol Imaging 2012:189254

41. Panizzi P, Swirski FK, Figueiredo JL, Waterman P, Sosnovik DE, Aikawa E, Libby P, Pittet M, Weissleder R, Nahrendorf M (2010) Impaired infarct healing in atherosclerotic mice with Ly-6C(hi) monocytosis. J Am Coll Cardiol 55:1629-1638 
42. Nahrendorf M, Sosnovik DE, Waterman P, Swirski FK, Pande AN, Aikawa E, Figueiredo JL, Pittet MJ, Weissleder R (2007) Dual channel optical tomographic imaging of leukocyte recruitment and protease activity in the healing myocardial infarct. Circ Res 100:1218-1225

43. Cai Y, Zhu L, Zhang F, Niu G, Lee S, Kimura S, Chen X (2013) Noninvasive monitoring of pulmonary fibrosis by targeting matrix metalloproteinases (MMPs). Mol Pharm 10:2237-2247

44. Korideck H, Peterson JD (2009) Noninvasive quantitative tomography of the therapeutic response to dexamethasone in ovalbumin-induced murine asthma. J Pharmacol Exp Ther 329:882-889

45. Cortez-Retamozo V, Swirski FK, Waterman P, Yuan H, Figueiredo JL, Newton AP, Upadhyay R, Vinegoni C, Kohler R, Blois J, Smith A, Nahrendorf M, Josephson L, Weissleder R, Pittet MJ (2008) Realtime assessment of inflammation and treatment response in a mouse model of allergic airway inflammation. J Clin Invest 118:4058-4066

46. Kossodo S, Zhang J, Groves K et al (2011) Noninvasive in vivo quantification of neutrophil elastase activity in acute experimental mouse lung injury. Int J Mol Imaging 2011:581406

47. Haller J, Hyde D, Deliolanis N, de Kleine R, Niedre M, Ntziachristos V (2008) Visualization of pulmonary inflammation using noninvasive fluorescence molecular imaging. J Appl Physiol (1985) 104:795-802

48. Stellari F, Sala A, Ruscitti F, Carnini C, Mirandola P, Vitale M, Civelli M, Villetti G (2015) Monitoring inflammation and airway remodeling by fluorescence molecular tomography in a chronic asthma model. J Transl Med 13:336

49. Chen WT, Mahmood U, Weissleder R, Tung CH (2005) Arthritis imaging using a near-infrared fluorescence folate-targeted probe. Arthritis Res Ther 7:R310-R317

50. Ryu JH, Lee A, Chu JU, Koo H, Ko CY, Kim HS, Yoon SY, Kim BS, Choi K, Kwon IC, Kim K, Youn I (2011) Early diagnosis of arthritis in mice with collagen-induced arthritis, using a fluorogenic matrix metalloproteinase 3specific polymeric probe. Arthritis Rheum 63:3824-3832
51. Li J, Ju Y, Bouta EM, Xing L, Wood RW, Kuzin I, Bottaro A, Ritchlin CT, Schwarz EM (2013) Efficacy of B cell depletion therapy for murine joint arthritis flare is associated with increased lymphatic flow. Arthritis Rheum 65:130-138

52. Peterson JD, Labranche TP, Vasquez KO et al (2010) Optical tomographic imaging discriminates between disease-modifying antirheumatic drug (DMARD) and non-DMARD efficacy in collagen antibody-induced arthritis. Arthritis Res Ther 12:R105

53. Wunder A, Schellenberger E, Mahmood U et al (2005) Methotrexateinduced accumulation of fluorescent annexin $\mathrm{V}$ in collagen-induced arthritis. Mol Imaging 4:1-6

54. Wunder A, Tung CH, Muller-Ladner U et al (2004) In vivo imaging of protease activity in arthritis: a novel approach for monitoring treatment response. Arthritis Rheum 50:2459-2465

55. Cho H, Bhatti FU, Yoon TW et al (2016) Non-invasive dual fluorescence in vivo imaging for detection of macrophage infiltration and matrix metalloproteinase (MMP) activity in inflammatory arthritic joints. Biomed Opt Express 7:1842-1852

56. Scales HE, Ierna M, Smith KM et al (2016) Assessment of murine collagen-induced arthritis by longitudinal non-invasive duplexed molecular optical imaging. Rheumatology (Oxford) 55:564-572

57. Abulrob A, Brunette E, Slinn J et al (2007) In vivo time domain optical imaging of renal ischemia-reperfusion injury: discrimination based on fluorescence lifetime. Mol Imaging 6:304-314

58. Amoozegar CB, Wang T, Bouchard MB et al (2012) Dynamic contrast-enhanced optical imaging of in vivo organ function. J Biomed Opt 17:96003-96001

59. Nakamura K, Tabata Y (2010) A new fluorescent imaging of renal inflammation with RCP. J Control Release 148:351-358

60. Ozaki M, Haga S, Ozawa T (2012) In vivo monitoring of liver damage using caspase-3 probe. Theranostics 2:207-214 